deed show promise in the therapy of autoimmune diseases. But if the interactions between mast cells and $\kappa$ light chains have such an important role in the effector phases of DTHR-as suggested here-the next question arises: What would be the side effects of neutralizing $\kappa$ light chains?

Mast cells were long considered to act almost exclusively as effector cells of IgEdependent allergic reactions. This viewpoint changed dramatically with studies showing that mast cells play a central role in innate immunity and T cell-mediated diseases, including contact hypersensitivity $^{8}$, psoriasis, rheumatoid arthritis ${ }^{7}$ and experimental allergic encephalitis (a model for multiple sclerosis $)^{14}$. If $\kappa$ light chains and mast cells have a similar role in initiating autoimmune diseases as for contact hypersensitivity, $\kappa$ light chains and mast cells might become new targets in the prevention of relapsing inflammatory autoimmune diseases.
1. Banchereau, J. \& Steinman, R.M. Dendritic cells and the control of immunity. Nature 392, 245-252 (1998).

2. Fuchs, E.J. \& Matzinger, P. B cells turn off virgin but not memory $T$ cells. Science 258, 56-59 (1992).

3. Qin, Z. et al. B cells inhibit induction of T cell-dependent tumor immunity. Nature Med. 4 627-630 (1998)

4. Tsuji, R.F. et al. Required early complement activation in contact sensitivity with generation of local C5-dependent chemotactic activity, and late T cell interferon $\gamma$ : A possible initiating role of B cells. J. Exp. Med. 186, 1015-1026 (1997).

5. Redegeld, F.A. et al. Immunoglobulin free light chains elicit immediate hypersensitivity-like responses. Nature Med. 8,694-701 (2002).

6. Wedemeyer, J., Tsai, M. \& Galli, S.J. Roles of mast cells and basophils in innate and acquired immunity. Curr. Opin. Immunol. 12, 624-631 (2000).

7. Stassen, M., Hültner, L. \& Schmitt, E. Classical and alternative pathways of mast cell activation. Crit. Rev. Immunol. (in the press).

8. Biedermann, T. et al. Mast cells control neutrophil recruitment during $\mathrm{T}$ cell-mediated delayed-type hypersensitivity reactions through tumor necrosis factor and macrophage inflammatory protein 2. J. Exp. Med. 192, 1441-1452 (2000).

9. Biedermann $T$, et al, Interleukin 4 instructs $T_{H} 1 \mathrm{re}$ sponses and resistance to Leishmania major in susceptible BALB/C mice. Nature Immunol. 2, 1054-1060 (2001).
10. Kunkel, E.J. \& Butcher, E.C. Chemokines and the tissue-specific migration of lymphocytes. Immunity 16, 1-4 (2002).

11. Ohashi, P.S. et al. Ablation of "tolerance" and in duction of diabetes by virus infection in viral antigen transgenic mice. Cell 65, 305-317 (1991).

12. Limmer, A. et al. Failure to induce organ-specific autoimmunity by breaking of tolerance: Importance of the microenvironment. Eur. J. Immunol. 28, 2395-2406 (1998).

13. McHale, J.F. et al. Vascular endothelial cell expression of ICAM-1 and VCAM- 1 at the onset of eliciting contact hypersensitivity in mice: Evidence for a dominant role of TNF- $\alpha$. /. Immunol. 162, 1648-1655 (1999).

14. Secor, V.H. et al. Mast cells are essential for early onset and severe disease in a murine model of multiple sclerosis. J. Exp. Med. 191, 813-821 (2000).

${ }^{1}$ Department of Dermatology and Allergology, Ludwigs-Maximilians-University

${ }^{2}$ Institute of Clinical Molecular Biology and Tumor Genetics

GSF-National Research Center for

Environment and Health

Munich, Germany

Email:mroecken@Irz.uni-muenchen.de or hueltner@gsf.de

\title{
Degenerate mice
}

The axons of motor neurons in humans can reach as long as three meters-that puts a big burden on the microtubule-based transport system that move molecules from the cell body out to axons. This system has been observed to break down in progressive degenerative motor-neuron diseases like amyotrophic lateral sclerosis (ALS). Indeed, decreased anterograde transport time occurs in mouse models of familial ALS. However, it is not clear if the defects in transport are a cause of the disease or a result of general cellular dysfunction.

In the 30 May issue of Neuron, LaMonte et al. directly test whether specific disruption of retrograde microtubule transport in spinal motor neurons of postnatal mice could recapitulate the motor-neuron degeneration of diseases like ALS. To do this, they disrupted the unidirectional microtubule motor composed of dynein and dynactin by overexpressing dynamitin, a subunit of dynactin. Previous studies had demonstrated that overexpression of dynamitin disassociates the micotubule binding and cargo-binding subunits of dynactin, effectively rendering it non-functional and disrupting dynein-mediated transport. The mice showed significant accumulation of neurofilaments and a decrease in retrograde transport times of a retrograde neurotracer, followed by late-onset progressive neurodegeneration remarkably similar to that of ALS. The mice developed muscular weakness, particularly in the hind legs, trembling, decreased stride length and endurance. As in many mouse ALS models the symptoms did not develop until 5-9 months of age and were variable similar to what occurs in sporadic ALS.

Shown are electron micrographs of the cross section of mouse ventral roots_bundles of axons leaving the spinal cord. In general, the large axons enervate fast-twitch muscle, and the small axons enervate slow-twitch muscle. On the left are sections from a wild-type mouse and on the right, a transgenic mouse. The transgenic mouse shows a tendency towards defects in axonal morphology. At this age (10-14 months) the wild-type and transgenic mice had a similar number of axons. But by 16 months the transgenic mice had

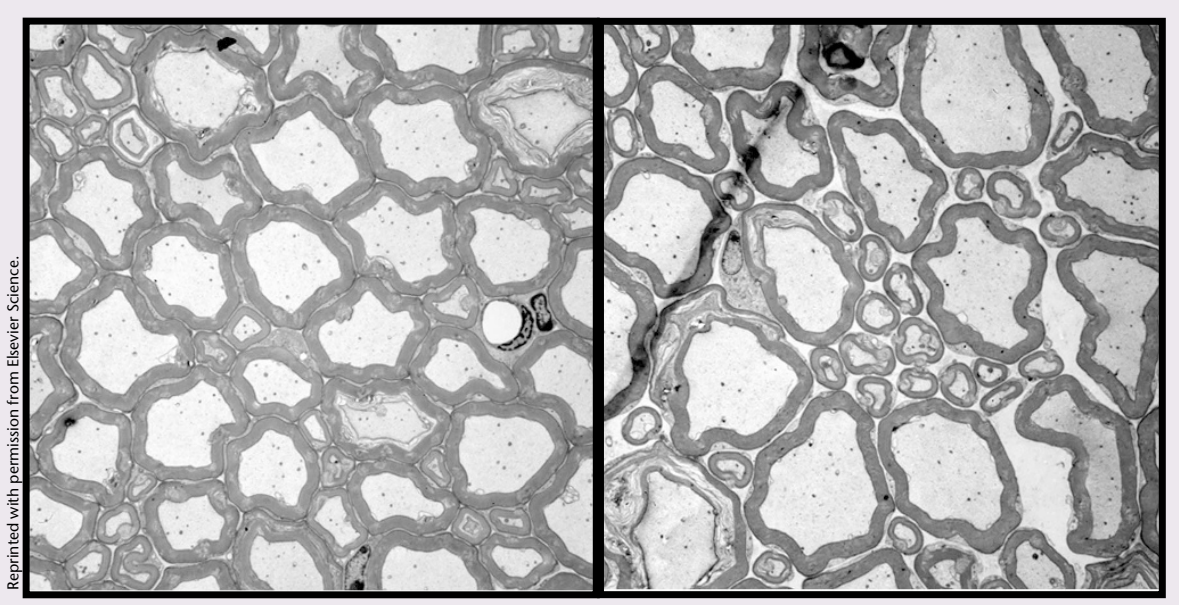
about 25 percent fewer axons, with the large caliber axons accounting for essentially all of the loss.

Although it had been suggested that defects in retrograde transport could be responsible for motor-neuron degeneration in these diseases, the hypothesis had not been formally tested. What remains now is to determine the mechanism of death. Toxicity from the accumulation of neurofilament seems like a likely candidate for this mechanism, but is by no means the only possible mode of disease progression.

Michael Stebbins 